\title{
Impedance Matching in Multi-Layer Interconnect Structures to Minimize Signal Reflections in High Speed Applications
}

\author{
Soorya Krishna K and M. S. Bhat
}

\begin{abstract}
Signal reflections due to impedance mismatch at via-interconnect junction is a major signal integrity issue in integrated circuits operating at Giga-Hertz $(\mathrm{GHz})$ frequencies. In this paper, we propose a method to reduce such via induced signal reflections in on-chip global interconnect lines. We show that the impedance matching can be achieved by the inclusion of an appropriate capacitive load at the junction of the on-chip interconnect line and via. Expressions to determine the capacitance value to be added at via-interconnect junction is derived. Simulation results show that the signal refection is reduced to less than $-10 \mathrm{~dB}$ in the frequency range of $1 \mathrm{GHz}$ to $10 \mathrm{GHz}$ using the proposed method in $65 \mathrm{~nm}$ technology. Proposed method is tested for two types of models - (i) Two interconnect layers connected through a single via and (ii) Two interconnect layers connected from layer 6 to layer 1 through five vias.
\end{abstract}

Index Terms-On-chip interconnects lines, signal reflection, via.

\section{INTRODUCTION}

Advancements in Very Large Scale Integrated Circuit (VLSI) technology has made it possible to incorporate more than eight metal layers connecting millions of components in a single Integrated Circuit (IC) chip [1]. Different interconnect layers are closely placed in an IC chip and vias are extensively used to connect different metal layers. As the data communication speed increases beyond 1 Giga Bits per Second (Gbps), signal integrity plays an important role in successful data transmission.

The impedance discontinuity at the junction of the via and on-chip global interconnect line results in signal reflections and contributes to the loss of the signal. To increase the efficiency in the propagation of the signal, designers try to eliminate every impedance mismatch along the high speed signal path because those discontinuities generate jitter, decrease the eye opening and maximum possible distance of data transmission. The improper connection of the vias between the two metal layers create considerable voltage breakdown, signal attenuation, crosstalk, switching noise etc. Studies on different types of vias, their properties and the impact on the propagation of signals are made in [2]-[5].

Electrical length of the vias is small and hence at lower frequencies of operation, vias are neglected or approximated

Manuscript received April 11, 2012; revised May 30, 2012.

$\mathrm{S}$. Krishna $\mathrm{K}$ is with the Department of Electronics and Communication Engineering, St. Joseph Engineering College, Vamanjoor-575028, India (e-mail: ksooryakrishna1@gmail.com)

M. S. Bhat is with the Department of Electronics and Communication Engineering, National Institute of Technology Karnataka, Surathkal, India (e-mail:msbhat@ieee.org). as lumped capacitance and inductance. When the frequency of signals transmitted through interconnects exceed $1 \mathrm{GHz}$, vias need to be modeled as distributed RLC structures. Further, due to scaling, technology node decreases but via resistance increases significantly $(0.3 \Omega$ in $0.7 \mu \mathrm{m}$ to $8 \Omega$ in $90 \mathrm{~nm})[6]$.

Different techniques are reported in the literatures to reduce the signal reflections in multi layer interconnect structures. The shape of the transmission line near via helps to reduce the signal reflections and is demonstrated in [7]. A new type of via structure is used in [8] to reduce the signal reflection in multi layer interconnect structures. In [9], signal reflections are reduced by optimizing via dimensions. In all these papers the reduction in signal reflection is achieved through the modification of via structure and it is aimed at printed circuit boards (PCBs). Further, in these cases the structure of the vias once fabricated remains fixed and modification of via structure is not feasible to suit newer frequencies of operation.

In this paper, we address the reduction of via induced signal reflection in ICs by incorporating simpler techniques such as inclusion of additional capacitance at via-interconnect junction for impedance matching. Such a technique is amenable to frequency tunability provided that the resulting capacitance value is derived from a digitally switchable bank of capacitors. In this case, the via impedance is calculated from its equivalent circuit and the effect on the signal reflections at the junction of the metal interconnect line and the via are analyzed for two types of models - (i) Two different interconnect metal layers connected through a single via and (ii) Two interconnect layers connected from layer 6 to layer 1 through five vias.

The rest of this paper is organized as follows. Introduction to via structure is given in section II. In section III, expression for the capacitance load to be included at via-interconnect junction for impedance matching is derived. Simulation results of two layer and six layer interconnect models using the proposed method is given in sections IV.A and IV.B respectively. Conclusions are given in section $\mathrm{V}$.

\section{Modeling OF THE VIAS}

Vertical Interconnect Access (VIA) makes vertical interconnections between wiring metal layers in IC chips [10]. In Deep Submicron (DSM) regime, via effects are considerable and cannot be neglected. In [11], via resistance $\left(R_{\text {via }}\right)$, via inductance $\left(L_{\text {via }}\right)$ and via capacitance $\left(C_{\text {via }}\right)$ are modeled as a function of physical dimensions and material characteristics of the via. A lumped $R L C$ model represented 
in the form of $\mathbf{T}$ network as the equivalent of a via is shown in Fig. 1. Voltage drop between the interconnected nodes of the two metal layers is caused by $R_{v i a}, L_{v i a}$, and capacitive component $C_{\text {via }}$ seen between via and ground. Expressions to estimate the resistance, inductance and capacitance values of the via equivalent circuit is given in equations 1,2 and 3 [11].

$$
\begin{gathered}
\mathrm{R}_{\mathrm{via}}=\frac{\rho \mathrm{h}_{\mathrm{via}}}{\pi \mathrm{V}_{\mathrm{via}}} \\
L_{v i a}=\frac{\mu_{0}}{4 \pi}\left[\begin{array}{l}
h_{v i a} \ln \left(\frac{2 h_{v i a}+\sqrt{r_{v i a}^{2}+\left(2 h_{v i a}\right)^{2}}}{r_{v i a}}\right)+ \\
\left(r_{v i a}-\sqrt{r_{v i a}^{2}+\left(2 h_{v i a}\right)^{2}}\right)
\end{array}\right] \\
C_{v i a}=\frac{2 \pi \varepsilon_{r} \varepsilon_{0} h_{v i a}}{\ln \left(\left(r_{v i a}+t\right) / r_{v i a}\right)}
\end{gathered}
$$

where $\rho$ is the resistivity of the conducting material, $r_{v i a}, h_{v i a}$, and $t$ represents the radius, length and dielectric thickness of the via respectively, $\varepsilon_{r}$ is the relative permittivity of the material, $\varepsilon_{0}$ is the permittivity of the free space $\left(8.852 \times 10^{-12}\right.$ $\mathrm{F} / \mathrm{m})$ and $\mu_{0}$ is the permeability of free space $\left(4 \pi \times 10^{-7} \mathrm{H} / \mathrm{m}\right)$.

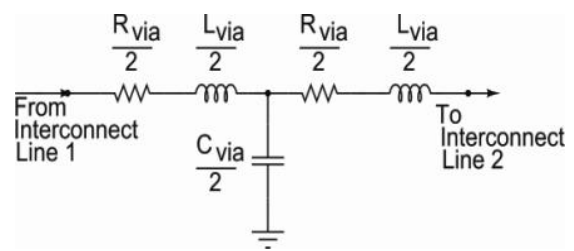

Fig. 1. Lumped RLC equivalent via model

\section{EXPRESSION TO FIND THE MATCHING LOAD CAPACITANCE VALUE}

Impedance discontinuity at the junction of the interconnect line and the via results in signal reflections. When the plane wave is propagating in the transverse electromagnetic (TEM) mode, the difference between the incident and reflected waves must be equal to the transmitted wave. i.e.

$$
\begin{gathered}
v_{t}=v_{i}-v_{r} \\
\frac{i_{t}}{Z_{v i a}}=\frac{i_{i}}{Z_{0}}-\frac{i_{r}}{Z_{0}}
\end{gathered}
$$

where $v_{i}, v_{r}, v_{t}, i_{i}, i_{r}$ and $i_{t}$ are the incident, reflected and transmitted voltage and current waves respectively. $Z_{0}$ is the characteristic impedance of the transmission line and $Z_{\text {via }}$ is the impedance of the via equivalent circuit. Expressions for the transmitted $\left(\mathrm{v}_{\mathrm{t}}\right)$ and reflected $\left(\mathrm{v}_{\mathrm{r}}\right)$ portions of the wave is obtained from equations (4) and (5) as,

$$
\begin{gathered}
v_{t}=v_{i} \frac{2 Z_{v i a}}{Z_{v i a}+Z_{0}} \\
v_{r}=v_{i} \frac{Z_{v i a}-Z_{0}}{Z_{v i a}+Z_{0}}
\end{gathered}
$$

The reflection coefficient $(\Gamma)$ is a measure of how much of the signal is reflected back by the intersection between the two impedance regions. From equations 6 and 7, we have

$$
\Gamma=\frac{v_{r}}{v_{i}}=\frac{Z_{v i a}-Z_{0}}{Z_{v i a}+Z_{0}}
$$

For implementation, interconnect metal lines are treated as microstrip lines and the characteristic impedance is given in Eq. 9 [13].

$$
Z_{0}=\sqrt{\frac{\varepsilon_{0} \mu_{0}}{\varepsilon_{e f f}}} \frac{1}{C_{a}}
$$

where

$$
\begin{gathered}
C_{a}=\left\{\begin{array}{l}
\frac{2 \pi \varepsilon_{0}}{\ln \left(\frac{8 h}{w_{e}}+\frac{w_{e}}{4 h}\right)} \quad \text { if } \frac{w_{e}}{h} \leq 1 \\
\varepsilon_{0}\left[\frac{w_{e}}{h}+1.393+0.667 \ln \left(\frac{w_{e}}{h}+1.444\right)\right] \text { if } \frac{w_{e}}{h} \succ 1
\end{array}\right. \\
w_{e}=\left\{\begin{array}{l}
w+0.398 t\left(1+\ln \frac{4 \pi w}{t}\right) \quad \text { if } \quad \frac{w}{h} \leq \frac{1}{2 \pi} \\
w+0.398 t\left(1+\ln \frac{2 h}{t}\right) \quad \text { if } \frac{w}{h} \succ \frac{1}{2 \pi} \\
\varepsilon_{\text {eff }}= \\
{\left[\frac{\varepsilon_{r}+1}{2}+\frac{\varepsilon_{r}-1}{2}\left(1+12 \frac{h}{w_{e}}\right)^{-\frac{1}{2}}+\zeta-0.217(\zeta-1) \frac{t}{\sqrt{w_{e} h}}\right]}
\end{array}\right.
\end{gathered}
$$

where $w, t$ and $h$ denotes the width, thickness of the conductor and substrate thickness respectively. The terms $e_{\text {eff }}$ and $w_{e}$ in Eq. 9 accounts for the effective dielectric constant and extra capacitance caused by the finite thickness of the signal conductor.

The input impedance $Z_{\text {in }}$ seen from the right terminal of the first interconnect layer (point B in Fig. 3) is computed by replacing the via in Fig. 3 by its equivalent circuit given in Fig. 1. Thus $Z_{\text {in }}$ is given by

$$
Z_{\text {in }}=\left(\frac{R_{v i a}+j \omega L_{v i a}}{2}\right)+\left[\frac{1}{j \omega C_{v i a}} / /\left(\begin{array}{l}
\frac{R_{v i a}+j \omega L_{v i a}}{2}+ \\
Z_{02}+\left(\frac{1}{j \omega C_{L}} / / Z_{t 2}\right)
\end{array}\right)\right]
$$

where $Z_{02}$ is the characteristic impedance of the second interconnect layer, $Z_{t 1}$ and $Z_{t 2}$ are the terminating resistances used for $S$ parameter analysis at the input and output side of the structure and $C_{L}$ is the load capacitance. Simplifying Eq. 10 , we get,

$$
Z_{\text {in }}=x+\frac{1}{x+Z_{02}+\frac{Z_{t 2}}{j \omega C_{L} Z_{t 2}+1}}
$$

where $x=\frac{R_{v i a}+j \omega L_{v i a}}{2}$. Signal reflection is minimum when the characteristic impedance of the microstrip line matches with the via impedance. The values of $R_{\text {via }}, L_{\text {via }}$ and $C_{\text {via }}$ for 65 $n m$ technology are computed using equations 1 to 3 and are found to be equal to $3.5 \Omega, 26.09 \mathrm{pH}$ and $113.98 \mathrm{fF}$ respectively.

For contemporary through silicon via architectures, the inductive voltage drop is found to exceed resistive voltage drop for frequencies above $3 \mathrm{GHz}$ [11] and hence via structure can be safely assumed to behave predominantly as 
an inductive load. In such a case, matching between the interconnect and the via can be easily achieved by connecting an appropriate matching capacitance between point B and ground as in Fig. 5. The new input impedance seen at point B in Fig. 5 is given by

$$
Z_{\text {match }}=\left(\frac{1}{j \omega C_{m}} / / Z_{\text {in }}\right)
$$

Substituting the expression for $Z_{\text {in }}$ in equation 12 and simplifying, expression for $\mathrm{C}_{\mathrm{m}}$ is given by

$$
C_{m}=\frac{1}{j \omega}\left[\frac{1}{Z_{\text {match }}}-\frac{1}{x+\frac{(a+j b)}{(c+j d+1)}}\right]
$$

where

$$
\begin{gathered}
x=\frac{R_{v i a}+j \omega L_{v i a}}{2} \\
a=R_{v i a}-\omega^{2} L_{v i a} C_{v i a} Z_{t 2}+2 Z_{02}+2 Z_{t 2} \\
b=R_{v i a} C_{L} Z_{t 2}+L_{v i a}+2 Z_{02} C_{L} Z_{t 2} \\
c=-\omega^{2} C_{v i a}\left(R_{v i a} C_{L} Z_{t 2}+L_{v i a}+2 Z_{02} C_{L} Z_{t 2}\right) \\
d=\omega C_{v i a}\left(R_{v i a}-\omega^{2} L_{v i a} C_{L} Z_{t 2}+2 Z_{02}+2 Z_{t 2}\right)
\end{gathered}
$$

The value of $C_{m}$ so obtained corresponds to optimum impedance matching and results in minimum signal reflection.

\section{Simulation Results}

Fig. 2 shows the 3D view of the two interconnect layers connected through a via. Modeling and simulations of the interconnect structures with via are carried out using Advanced Digital System (ADS) software. In this work, we have used interconnect parameters of $65 \mathrm{~nm}$ technology [12].

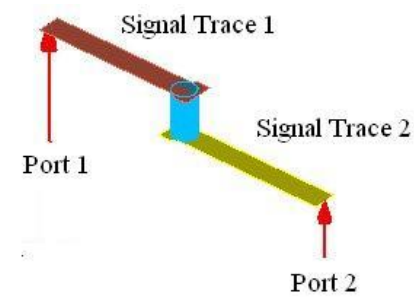

Fig. 2. 3D view of the two interconnect layers connecting through via

\section{A. Model of Top Two Interconnect Layers Connected Through a Via}

We have considered the model of two different interconnect metal layers corresponding to the top two layers in the multi layer interconnect structure which are connected through a via. For the simulation, following via dimensions are considered: via diameter of $0.4 \mu \mathrm{m}$, pad diameter of 0.65 $\mu \mathrm{m}$, via height of $0.6 \mu \mathrm{m}$ and dielectric thickness $1 \mu \mathrm{m}$. A transmitter and a receiver are connected using such an interconnect. The receiver section is modeled as load capacitance $C_{L}$. Interconnect line length and $C_{L}$ are considered as $0.5 \mathrm{~mm}$ and $0.25 \mathrm{pF}$ respectively.

To know the effect of via, signal reflections between two interconnect layers connected without and with via are measured. Figure 3 shows the ADS setup for the two interconnect layers connected through a via and signal reflection is measured at point A. From Fig. 4, it can be seen that upto a frequency of $9 \mathrm{GHz}$, there is a significant increase in the signal reflection in the structure employing via in comparison to the structure without via. This is due to the impedance mismatch between the interconnect layer and via.

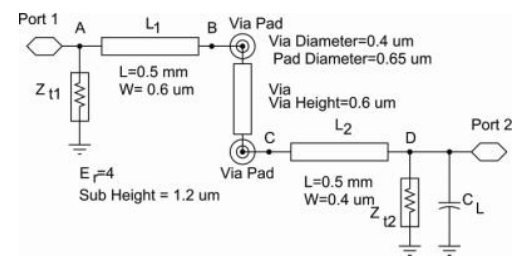

Fig. 3. Structure of the two interconnect layers connected through a via

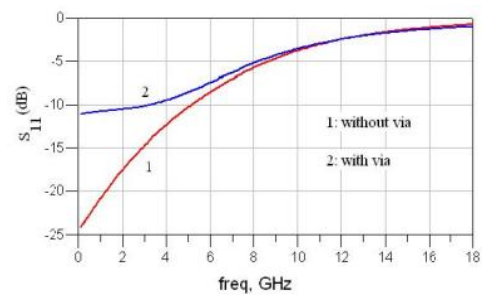

Fig. 4. Signal reflection with and without via.

To mitigate the signal reflection at the junction of the via and the interconnect; a capacitance is inserted between point $\mathrm{B}$ and ground as shown in Fig. 5. Figure 6 shows the signal reflections at the input side (point $\mathrm{A}$ in Fig. 5) for different capacitance $\left(C_{m}\right)$ values.

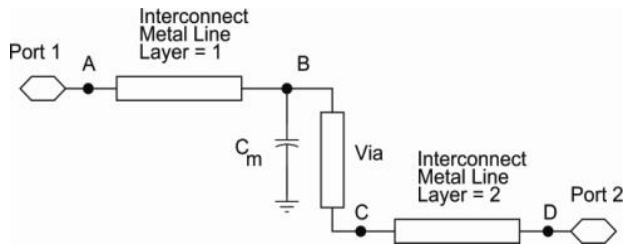

Fig. 5. Model used to measure the signal reflection in two interconnect layers connected through a via

It has been observed that the minimum signal reflection point is dependent on the matching terminating resistances at the input $\left(Z_{t 1}\right)$ and output side $\left(Z_{t 2}\right)$ of the structure and the matching capacitance $C_{m}$. In this work, we are interested to know the signal reflections in the range of 1-10 $\mathrm{GHz}$. By keeping the minimum signal reflection point at $5 \mathrm{GHz}$ with $Z_{t I}=76 \Omega$ and $Z_{t 2}=88 \Omega$, the theoretical value (Eq. 13) of $C_{m}$ is found to be $345 \mathrm{fF}$. From the simulations, the matching capacitance value is found to be $331 \mathrm{fF}$ at $5 \mathrm{GHz}$ (Fig. 6).

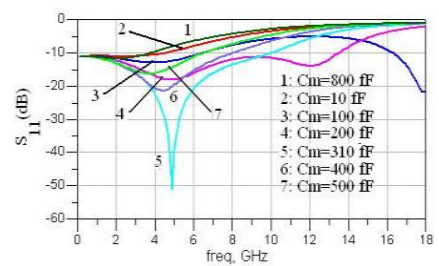

Fig. 6. Signal reflection for different matching capacitance values.

Fig. 7 shows the signal reflection $\left(S_{11}\right)$ at point A with and 
without matching capacitance at point B in Fig. 5. From Fig. 7, it can be seen that there is a clear advantage in using a matching capacitance at the junction of the metal interconnect layer and the via to reduce signal reflections in the frequency range of $1-10 \mathrm{GHz}$.

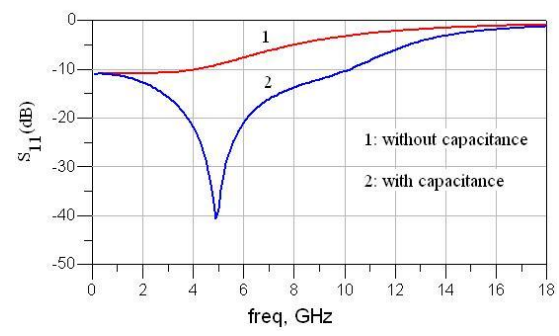

Fig. 7. Simulation result of signal reflection with and without capacitance

\section{B. Model of a Six Layer Structure}

Analysis of the proposed method is made for a six layer interconnect structure in which the two interconnect metal lines are connected from layer 6 to layer 1 through five vias. The ADS setup for creating six layer structures is given in Fig. 8. The values of the relative dielectric constant of the substrate $\left(\varepsilon_{\mathrm{r}}[\mathrm{n}]\right)$, height of substrate $(\mathrm{H}[\mathrm{n}])$, dielectric loss tangent $(\mathrm{TanD}[\mathrm{n}])$, metal thickness $(\mathrm{T}[\mathrm{n}])$, conductivity (Cond[n]), type of the metal layer (LayerType[n]) and name (LayerName[n]) are listed in Fig. 8.

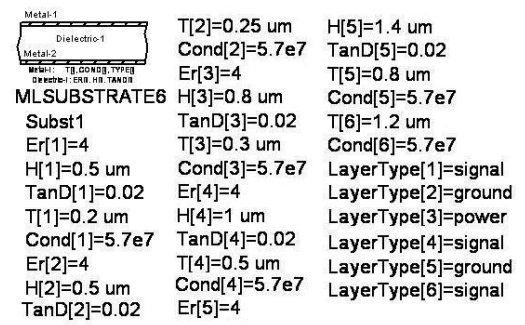

Fig. 8. ADS setup for the simulation of 6 layer structure

Capacitances are added at the junction of each interconnect layer and via. The arrangement for the connection of two interconnects lines from layer 6 to layer 1 through vias is shown in Fig. 9. Vias are connected with suitable dimensions which are matching with the width of the corresponding interconnect layers.

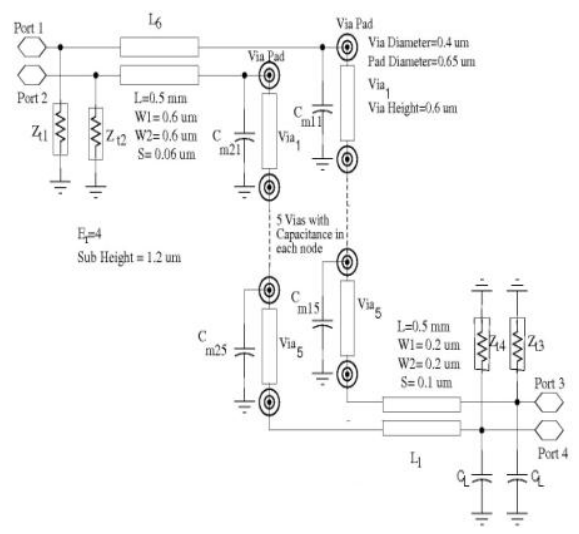

Fig. 9. Interconnect layer 6 is connected to layer 1 through 5 vias with capacitance at each junction.

Fig. 10 shows the signal reflection with and without capacitance in a six layer structure. It can be seen that, there is significant improvement in signal reflection with the addition of the matching capacitance at the junction of each interconnect layer and the via.

Using the proposed method, signal reflections can be reduced in a multi layer structures. In multi layer structures, signal lines are surrounded by ground/power lines for reducing crosstalk. Hence the matching capacitances can be easily connected between the ground plane and the junction of the interconnect layer and the via. The matching capacitances $\left(C_{m}\right)$ can be realized using MOS devices. Since the value of $C_{m}$ is moderate, the area consumed by these capacitors will not be very significant.

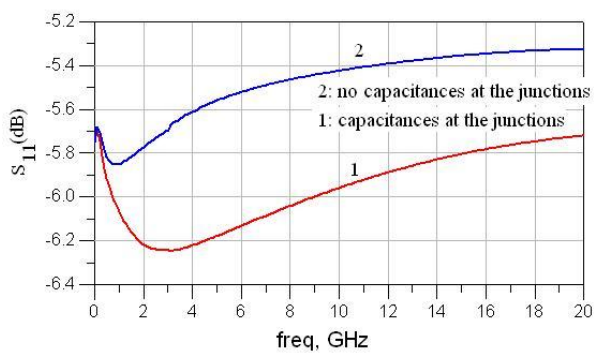

Fig. 10. Signal reflection with and without capacitance in six layer structure

\section{Scope for the Frequency Tunable Signal Reflection} Reduction

We propose a method to reduce signal reflection at the junction of the metal interconnect layer and via for different frequencies of operation. Figure 11 shows the arrangement of a frequency tunable signal reflection reduction mechanism for the two interconnect layer structure connected through a single via. A bank of capacitors $\left(C_{m l}\right.$ to $\left.C_{m n}\right)$ are connected using a set of switches $\left(S_{1}\right.$ to $\left.S_{n}\right)$ in place of the single matching capacitance at the junction of the metal layer and the via. Depending upon the frequency of operation, digitally controlled switches are turned ON or OFF so as to present right value of capacitance at the interconnect-via junction for impedance matching.

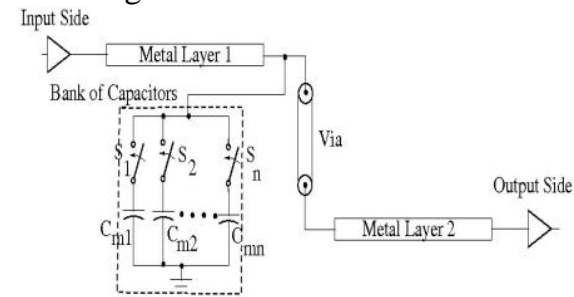

Fig. 11. Arrangement for the frequency tunable signal reflection reduction.

Although, parasitic capacitances of the switches need to be taken into account while computing the optimum matching capacitance to minimize signal reflection for a given frequency, the method helps in reducing the reflection for any frequency of operation. Switches can be realized using MOS devices.

\section{CONCLUSION}

$\mathrm{S}$ parameter analysis of the two interconnect layer model connected with and without via shows that signal reflection increases with the inclusion of via structure. This is due to the impedance mismatch at the junction of the via and the metal 
interconnect line. In this paper, we have proposed a method to reduce the signal reflection by adding a matching capacitance at the via-interconnect junction. The matching capacitance value is analytically computed and is found to be in close agreement with the simulation results. In the two layer interconnect model, signal reflections are reduced to less than $-10 \mathrm{~dB}$ in the frequency range of $1 \mathrm{GHz}$ to $10 \mathrm{GHz}$. The proposed method is also applied to six layer interconnect structure and the signal reflection is found to reduce considerably. The above method can be extended to different operating frequencies by incorporating digitally tunable matching capacitances.

\section{ACKNOWLEDGMENT}

The authors would like to thank the Department of Information Technology, MCIT, and Govt. of India for providing financial support to carry out this work under SMDP VLSI project.

\section{REFERENCES}

[1] Interconnect Technology Report, www: itrs.net, 2007

[2] H. Chen, Q. Li, and L. Tsang, "Analysis of a Large Number of Vias and Differential Signaling in Multilayer Structures," IEEE Transactions on Microwave Theory and Techniques, vol. 51, no. 3, pp. 818-829, 2003.

[3] P. Kok and D. D. Zutter, "Capacitance of a Circular Symmetric Model of a Via Hole including Finite Ground Plane Thickness," IEEE Trans. Microwave Theory Tech, vol. 39, pp. 1229-1234, 1999.

[4] P. A. Kok and D. D. Zutter, "Scalar Magneto Static Potential Approach to the Prediction of the Excess Inductance of Grounded Via's and Via's through a Hole in a Ground Plane," IEEE Trans. on Microwave Theory Tech, vol. 42, pp. 1229-1237, 1994.

[5] J. P. Quine, H. F. Webster, H. H. Glascock, and R. O. Carlson. "Characterization of Via Connections in Silicon Circuit Boards," IEEE Trans. on Microwave Theory Tech., vol. 36, pp. 21-27, 1998.

[6] E. Sicard and S. D. Bendhia, Basics of CMOS Cell Design, McGraw-Hill Publication, 2007.

[7] D. H. Kwon, J. Kim, K. Kim, S. C. Choi, J. Lim, J. H. Park, L. Choi, S. W. Hwang, and S. H. Lee, "Characterization and Modelling of a New Via Structure in Multi layered Printed Circuit Boards," IEEE Transactions on Components and Packaging Technologies, vol.26, no. 2, pp. 483-490, 2003.

[8] Y. W. Kim, J. H. Kim, H. W. Yang, and O. K. Kwon, "A New Via Hole Structure of MLB for RF and High Speed Systems," Electronic
Components and Technology Conference, vol. 55, pp. 1378-1382, 2005.

[9] W. Y. Chang, Richard, K. Y. See, and E. K. Chua, "Comprehensive Analysis of the Impact of Via Design on High-Speed Signal Integrity," 9th Electronics Packaging Technology Conference, pp. 262-267, 2007.

[10] S. H. Hall, G. W. Hall, and J. A. M. Call, High Speed Digital System Design - A handbook of Interconnect Theory and Design Practice, Wiley Publication, 2000.

[11] G. Katti, M. Stucchi, K. D. Meyer, and W. Dehaene, "Electrical Modeling and Characterization of Through Silicon via for Three-Dimensional ICs," IEEE Transactions on Electron Devices, vol. 57, no. 1, pp 256-262, 2010.

[12] P. Bai, C. Auth, S. Balakrishnan, and M. Bost, Brain et al, "A 65 nm Logic Technology Featuring $35 \mathrm{~nm}$ Gate Lengths, Enhanced Channel Strain, $8 \mathrm{Cu}$ Interconnect Layers, Low-k ILD and $0.57 \mu \mathrm{m}^{2}$ SRAM Cell," TEDM 2004 Conference Proceedings, pp. 657-660, 2004

[13] S. H. Hall and H. L. Heck, "Advanced Signal Integrity for High Speed Digital Designs,” Wiley Publication, 2009.

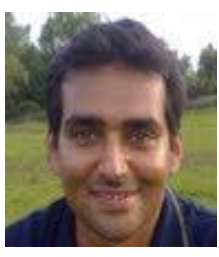

Soorya Krishna K received his B.E in Electronics and Communication Engineering from Kuvempu university and M.Tech from V.T.U. Belgaum Presently pursuing $\mathrm{PhD}$ in the department of Electronics and Communication Engineering in NITK Surathkal. He is working as an Assistan Professor in the Department of Electronics and Communication in St. Joseph Engineering College, Vamanjoor. His research interests include High Speed Interconnect design, Low Power VLSI design.

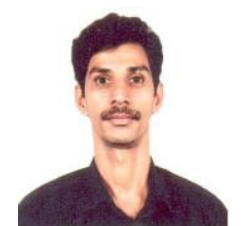

M S Bhat received his M.Sc. in Physics from Mangalore University, M.Tech from KREC Surathkal and $\mathrm{PhD}$ from Indian Institute of Science, Bangalore in 1987, 1989 and 2007 respectively. He joined the Department of Electronics and Communication Engineering, NITK Surathkal as a faculty in 1989 and currently, he is a Professor in the department. At NITK Surathkal, he was the coordinator of World Bank Sponsored project IMPACT in 2000-2002. Since 2007, he is the Co-coordinator of Special Manpower Development Project in VLSI, funded by Department of Information Technology, Govt. of India Currently he is supervising 2 projects in the area of RF MEMS circuits funded by DIT and NPMASS, Govt. of India. His research interests include High Speed Interconnect Design, RF MEMS Circuit Design, Low Power VLSI Design and Image \& Signal Processing. 